\title{
Fracture Loci in Sheet Metal Forming: A Review
}

\author{
M. Beatriz Silva ${ }^{1} \cdot \operatorname{Kerim~Isik~}^{2} \cdot$ A. Erman Tekkaya ${ }^{2}$ Paulo A. F. Martins ${ }^{1}$
}

Received: 11 June 2015/Revised: 15 October 2015/Published online: 27 November 2015

(C) The Chinese Society for Metals and Springer-Verlag Berlin Heidelberg 2015

\begin{abstract}
Fracture in sheet metal forming usually occurs as ductile fracture, rarely as brittle fracture, at the operating temperatures and rates of loading that are typical of real processes in two different modes: (1) tensile and (2) in-plane shear (respectively, the same as modes I and II of fracture mechanics). The circumstances under which each mode will occur are identified in terms of plastic flow and ductile damage by means of an analytical approach to characterize fracture loci under plane stress conditions that takes anisotropy into consideration. Fracture loci was characterized by means of the fracture forming limit line and by the shear fracture forming limit line in the fracture forming limit diagram. Experiments were performed with single point incremental forming and double-notched test specimens loaded in tension, torsion and in-plane shear give support to the presentation and allow determining the fracture loci of AA1050-H111 aluminium sheets with $1 \mathrm{~mm}$ thickness. The relation between fracture toughness and the fracture forming limits was also investigated by comparing experimental values of the strains at fracture obtained from a truncated conical part produced by single point incremental forming and from double-notched test specimens loaded in tension.
\end{abstract}

\section{KEY WORDS: Sheet forming; Fracture; Fracture forming limit diagram}

\section{Introduction}

Formability in sheet metal forming sets limits on the amount of deformation that can be imparted to sheet metal blanks without failure by wrinkling, necking or fracture. Marciniak [1] was the first researcher to incorporate these limits in the principal strain space by considering a formability limit by wrinkling, a formability limit by necking and a formability limit by fracture (Fig. 1a).

Available online at http://link.springer.com/journal/40195

Paulo A. F. Martins

pmartins@ist.utl.pt

1 IDMEC, Instituto Superior Técnico, Universidade de Lisboa, Av. Rovisco Pais, 1049-001 Lisbon, Portugal

2 Institute of Forming Technology and Lightweight Construction, Technical University of Dortmund, Baroper Str. 303, 44227 Dortmund, Germany
The formability limit by wrinkling is located in the lower left-hand part of the second quadrant and is influenced by many factors such as the mechanical properties of the material, the geometry of the sheet metal part, the contact conditions imparted by the tools and the applied level of stresses and strains. The difficulty in combining all these factors into a universal criterion influenced the investigation of wrinkling to be carried out case by case for specific sheet metal forming processes. Kim and Yang [2], for example, provide a comprehensive overview of the published literature in the field and proposed an energybased criterion to determine the onset of wrinkling in various sheet metal forming processes such as cylindrical, spherical and elliptical cup deep drawing.

The formability limit by necking is characterized by a 'V-shaped' curve [designated as the forming limit curve (FLC)] that indicates the amount of deformation where aesthetics problems and incipient fracture derived from localized zones of thinning are likely to develop in sheet 
(a)

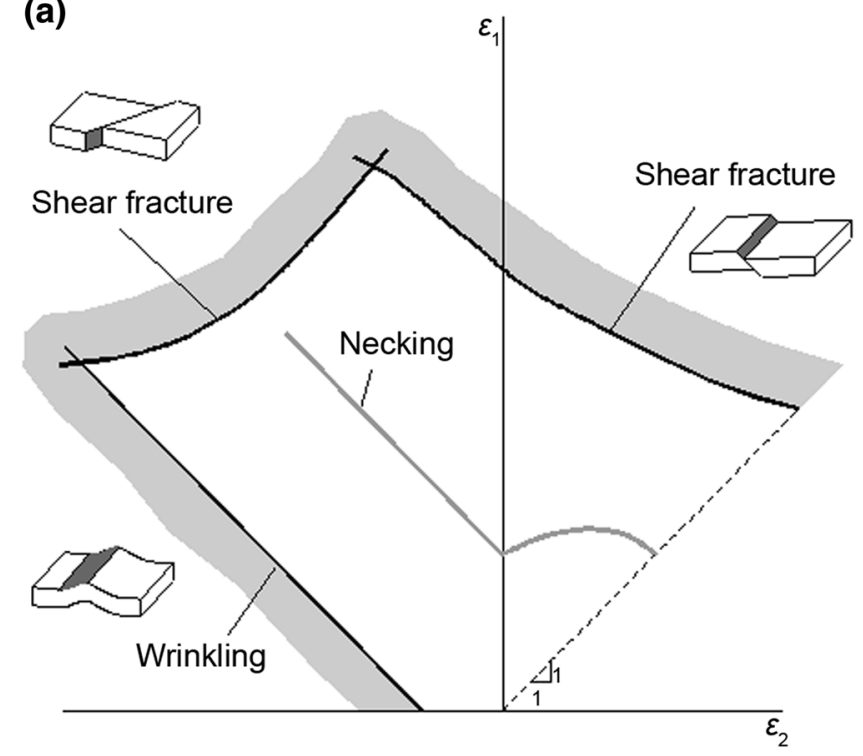

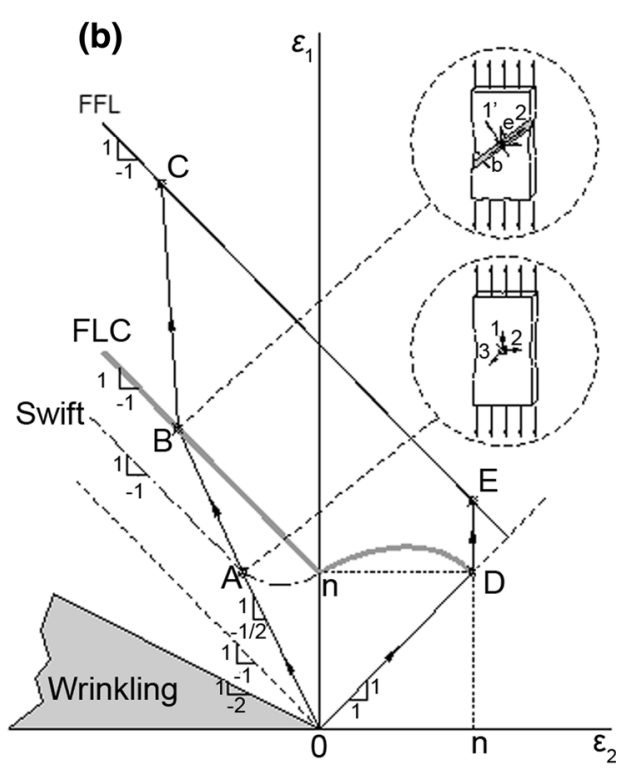

Fig. 1 Formability limits of sheet metal forming in the principal strain space: a Marciniak's vision [1]; b schematic representation of the forming limit curve (FLC) and of the fracture forming limit line (FFL)

metal parts. When the evolution of experimental in-plane strains under different loading paths is plotted in the principal strain space, the locus of strain pairs at which localized necking occurs is called the forming limit diagram (FLD) which was originally proposed by Keeler [3] for the tension-tension domain and extended by Goodwin [4] for the tension-compression domain.

In the tension-compression (left-hand) quadrant, plane stress plasticity theory predicts the in-plane strain pairs at which diffuse [5] and localized necks [6] occur and the angles with respect to the major loading axis at which localized necks form. In the tension-tension quadrant, theory predicts that diffuse necks will occur, but there is no continuum theory to explain the occurrence of localized necks that experiments show usually to form perpendicular to the greatest tensile strain. This led Marciniak and Kuczynski [7] to postulate the existence of locally thinned regions in the sheets at which necks initiate.

The formability limit by fracture consists of two curves (designated as the fracture locus) that intersect at the upper right-hand part of the second quadrant and delimit the strain loading conditions where cracks are triggered. In Marciniak's original vision [1], fracture occurs by in-plane or out-of-plane (through-thickness) shear stresses but to the author's knowledge, this assumption and its corresponding fracture loci depicted in Fig. 1a were never accompanied by any phenomenological model or experimental evidence.

The relation between the formability limits by necking and fracture is schematically plotted in Fig. 1b, where typical strain loading paths experience sharp changes towards plane strain deformation after crossing the FLC (refer to the loading paths OABC and ODE). This is because after necking, a sheet reduces in thickness at the site of neck initiation and spreads longitudinally in plane strain. The FLC may, therefore, be regarded as the locus of all the in-plane strains where sharp changes in loading path occur since all prior loading paths become plane strain $\left(\mathrm{d} \varepsilon_{2}=0\right)$ after necking.

Atkins [8] showed that the uppermost fracture locus falling from left to right can be associated with both the condition of critical thickness reduction at failure and the ductile fracture criterion due to McClintock's research [9] and proposed its graphical representation as a straight line (designated as the fracture forming limit line (FFL)) with a slope equal to -1 . The ductile fracture criterion due to McClintock' work [9] is based on the stress triaxiality ratio $\sigma_{\mathrm{H}} / \bar{\sigma}$ (defined as the ratio of the average and the effective stress) and is known to play an important role in the analysis of the formability of metals based on void growth models. In the same year, Muscat-Fenech et al. [10] correlated the FFL with fracture toughness in mode I and concluded that the fracture locus given by the FFL corresponds to crack opening by tension instead of crack opening by out-of-plane shear (mode III of fracture mechanics), as it was originally suggested by Marciniak [1].

Since the mid-1990s, there have been several alternative proposals for the formability limits by fracture. Special emphasis is given to the work of Wierzbicki et al. [11], who proposed new fracture models that combine conditions of stress triaxiality $\sigma_{\mathrm{H}} / \bar{\sigma}$, Lode angle parameters and deviatoric stresses with material-dependent fitting 


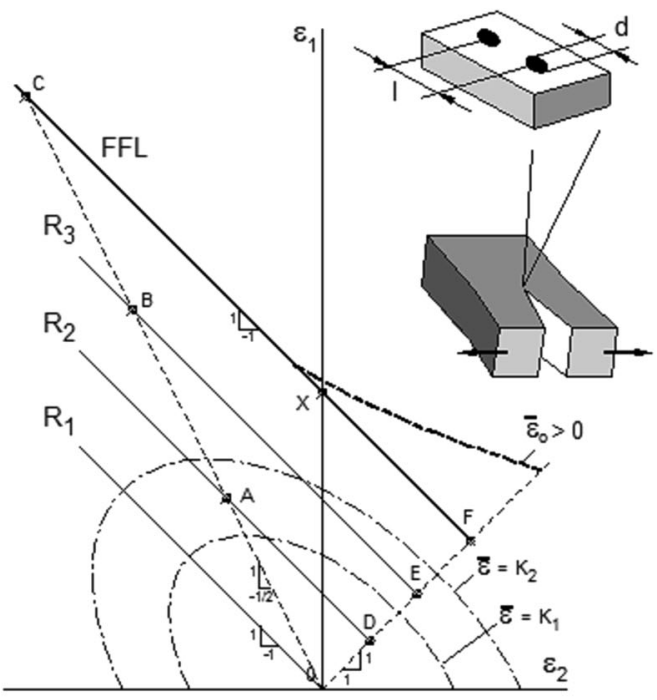

(a)

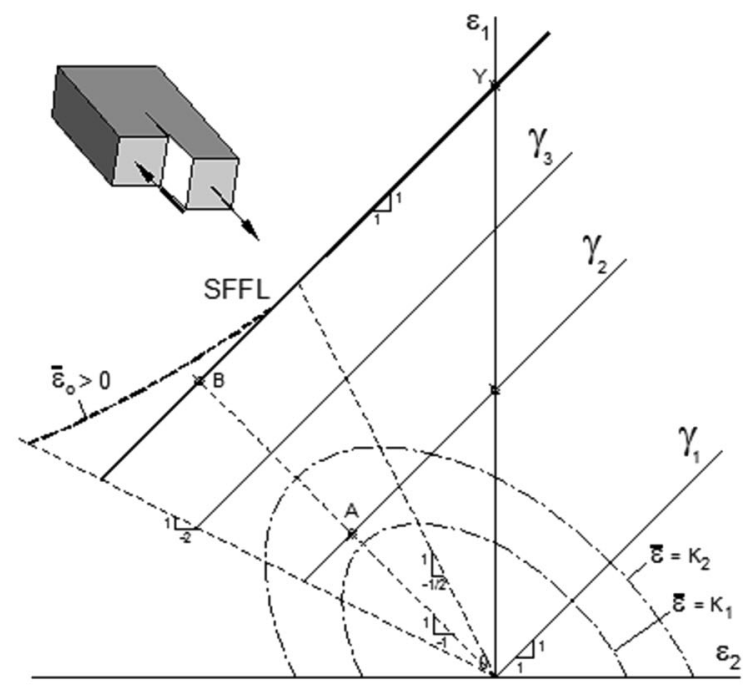

(b)

Fig. 2 Schematic representation of the fracture forming limit line (FFL) a and in-plane shear fracture forming limit line (SFFL) b in the principal strain space

procedures to establish alternative shapes to the formability limits by fracture in the principal strain space and in the space of the effective strain at fracture versus stress triaxiality.

More recently, Isik et al. [12] proposed a new vision for the formability limits by fracture in sheet metal forming that extended the findings of Atkins on the FFL [8] by introducing an in-plane shear fracture forming limit line (SFFL) on the basis of the critical values of distortion $\gamma$ and the maximum allowable plastic shear work per unit of volume $\int \tau \mathrm{d} \gamma$ at the onset of fracture. This new vision was supported by an analytical framework aimed at providing understanding to the circumstances under which cracking occurs in terms of plastic flow and ductile damage and by experimentation focused on the determination of the fracture strain pairs.

This paper draws from the analytical framework that was recently proposed by Isik et al. [12], which demonstrates that plastic flow and failure in sheet metal forming results from competition between modes I and II of fracture mechanics, to the determination of the fracture loci of AA1050-H111 aluminium sheets by a wide range of experimental tests that include double-notched test specimens loaded in tension, torsion and in-plane shear and single point incremental forming of truncated conical and pyramidal test geometries. A link between crack opening by mode $\mathrm{I}$ in double-notched test specimens loaded in tension and SPIF of truncated conical parts is also utilized to discuss some of the reasons why FFLs (or SFFLs) instead of FLCs should be considered material properties.

\section{Theory}

At the operating temperatures and rates of loading that are typical of real sheet metal forming processes, fracture usually occurs as ductile fracture, rarely as brittle fracture, in two different opening modes: (1) tensile and (2) in-plane shear (respectively, the same as modes I and II of fracture mechanics). The circumstances under which each mode will occur are identified in terms of plastic flow and microstructural ductile damage by means of an analytical framework to characterize fracture loci under plane stress conditions that takes anisotropy into consideration.

\subsection{Mode I: Tensile Fracture}

Irrespective of the initial loading history before necking, tensile fracture occurs approximately at a constant throughthickness true strain $\varepsilon_{3 \mathrm{f}}$ corresponding to a constant percentage of the reduction in thickness at fracture $R_{\mathrm{f}}$ given by $\left(t_{0}-t_{\mathrm{f}}\right) / t_{0}$ where $t_{0}$ is the initial thickness of the sheet and $t_{\mathrm{f}}$ is the thickness at fracture. The reduction in thickness at fracture $R_{\mathrm{f}}$ and $\varepsilon_{3 \mathrm{f}}$ is related by $\varepsilon_{3 \mathrm{f}}=\ln \left(1-R_{\mathrm{f}}\right)$.

Owing to constancy of volume $\varepsilon_{1 \mathrm{f}}+\varepsilon_{2 \mathrm{f}}+\varepsilon_{3 \mathrm{f}}=0$ during plastic flow, it follows that the FFL is a straight line falling from left to right with slope of -1 in the principal strain space (refer to Fig. 2a where lines of constant $R_{\mathrm{f}}$ are shown). Figure $2 \mathrm{a}$ also presents two proportional loading paths (OC and $\mathrm{OF}$ ) corresponding to uniaxial tension and equal biaxial stretching that fail by fracture at $\mathrm{C}$ and $\mathrm{F}$, respectively. For the purpose of simplifying the presentation, both loading paths are taken as linear up to the onset 
of fracture, without experiencing the change in direction towards plane strain conditions that one would expected to occur after crossing the FLC (refer to Fig. 1b).

Considering the modification of the effective strain fracture criterion $\bar{\varepsilon}=K$ by means of a non-dimensional weighting function built upon the stress triaxiality ratio $\sigma_{\mathrm{H}} / \bar{\sigma}$ of the hydrostatic $\sigma_{\mathrm{H}}$ and effective $\bar{\sigma}$ stresses, it is possible to write the following damage criterion,

$D_{\text {crit }}=\int_{0}^{\bar{\varepsilon}_{\mathrm{f}}} \frac{\sigma_{\mathrm{H}}}{\bar{\sigma}} \mathrm{d} \bar{\varepsilon}$.

This criterion is related to the original work of McClintock [9] and its critical value $D_{\text {crit }} \propto \ln (l / d)$ may be formulated in terms of the microstructural void parameters that relate the inter-hole $l$ (inter-particle inclusion) spacing and the average diameter $d$ of the holes (particles) (Fig. 2a) [13].

Martins et al. [14] showed that by using the constitutive equations associated with Hill's 1948 anisotropic yield criterion and assuming rotational symmetry anisotropy $r_{\alpha}=r=\bar{r}$, where $\bar{r}$ is the normal anisotropy, it is possible to rewrite Eq. (1) as a function of the major and minor inplane strains $\left(\varepsilon_{1 \mathrm{f}}, \varepsilon_{2 \mathrm{f}}\right)$ at the onset of fracture,

$D_{\text {crit }}=\int_{0}^{\varepsilon_{1 \mathrm{f}}} \frac{(1+r)}{3}\left(\frac{\beta+1}{\beta}\right) \mathrm{d} \varepsilon_{1}=\frac{(1+r)}{3}\left(\varepsilon_{1 \mathrm{f}}+\varepsilon_{2 \mathrm{f}}\right)$,

where $\beta=\mathrm{d} \varepsilon_{1} / \mathrm{d} \varepsilon_{2}$ is the slope of a general proportional strain path. It follows from Eq. (2) that the critical value of the damage criterion $D_{\text {crit }}$ also defines a straight line with slope of -1 falling from left to right in close agreement with the FFL and the condition of critical reduction of thickness at fracture.

Three additional conclusions are extracted from Eq. (2). Firstly, the integrand has the form $(A+B / \beta)$, implying that the damage function for a constant strain ratio $\beta$, is independent of the loading path history. This occurrence is discussed by Atkins and Mai [13] and justifies the reason why strain loading paths in Fig. 2a were assumed as linear. Secondly, if the lower limit of the integral in Eq. (2) is $\bar{\varepsilon}_{0}$ rather than zero, corresponding to situations where there is a threshold strain $\bar{\varepsilon}_{0}$ below which damage is not accumulated, the FFL deviates from a straight line and presents the 'upward curvature' that is schematically represented by the dashed solid line in Fig. 2a [14]. Thirdly, combining the relation between the FFL and fracture toughness in mode I that was originally proposed by Muscat-Fenech et al. [10] with above mentioned conclusions regarding the critical reduction in thickness $R_{\mathrm{f}}$ and the critical ductile damage $D_{\text {crit }}$ being constant and independent from deformation history up to fracture, it follows that the FFL is a material property in contrast to the FLC that depends on the strain loading path.

\subsection{Mode II: Shear Fracture}

In what regards crack opening by in-plane shear (mode II of fracture mechanics), it is important to understand that straight lines $\gamma_{1}, \gamma_{2}$ and $\gamma_{3}$ rising from left to right and corresponding to maximum values of the in-plane distortion $\gamma_{12}$ in the Mohr's circle of strains have slope of +1 and are perpendicular to the FFL (Fig. 2b). In-plane distortions $\gamma_{12}$ (hereafter designated as $\gamma$ ) are caused by in-plane shear stresses $\tau_{12}$ (hereafter designated as $\tau$ ) and, therefore, it is likely that the in-plane shear fracture limiting locus (SFFL) will coincide with a straight line of slope equal to +1 , in which the major and minor inplane strains and distortions take critical values at fracture, $\varepsilon_{1 \mathrm{f}}-\varepsilon_{2 \mathrm{f}}=\gamma_{\mathrm{f}}$, where, $\gamma_{\mathrm{f}}=Y$ (Fig. 2b). Thus, if the weighting function that corrects the accumulated value of the effective strain until fracture $\bar{\varepsilon}_{\mathrm{f}}$ as a function of the strain loading paths is taken as the in-plane shear stress ratio $\tau / \bar{\sigma}$ instead of the stress triaxiality ratio $\sigma_{\mathrm{H}} / \bar{\sigma}$ it is possible to define the following damage criterion [14],

$$
\begin{aligned}
D_{\text {crit }}^{\mathrm{s}} & =\int_{0}^{\bar{\varepsilon}_{\mathrm{f}}} \frac{\tau}{\bar{\sigma}} \mathrm{d} \bar{\varepsilon}=\int_{0}^{\bar{\varepsilon}_{1 \mathrm{f}}} \frac{1}{2} \frac{(1+r)}{(1+2 r)}\left(\frac{\beta-1}{\beta}\right) \mathrm{d} \varepsilon_{1} \\
& =\frac{1}{2} \frac{(1+r)}{(1+2 r)}\left(\varepsilon_{1 \mathrm{f}}-\varepsilon_{2 \mathrm{f}}\right) .
\end{aligned}
$$

The critical values of damage by in-plane shear $D_{\text {crit }}^{\mathrm{s}}$ derived from Eq. (3) are located along a straight line rising from left to right with a slope equal to +1 in agreement with the condition of critical distortion $\gamma_{\mathrm{f}}$ along the SFFL. By following a procedure similar to that performed for the FFL in case the lower limit of the integral in Eq. (3) is $\bar{\varepsilon}_{0}$, it is also possible to conclude that the SFFL deviates from a straight line and presents an 'upward curvature' illustrated by the dashed solid line in Fig. 2 b.

\section{Experimental}

\subsection{Mechanical Characterization of the Material}

The investigation was carried out in AA1050-H111 aluminium sheets with $1 \mathrm{~mm}$ thickness. The mechanical characterization of the material at room temperature was performed by means of tensile tests on an INSTRON 4507 universal testing machine. The tests followed the ASTM standard E8/E8 M [15], and the resulting average stressstrain curve was approximated by the following LudwikHollomon's equation, 
$\sigma=140 \varepsilon^{0.04}$.

Table 1 provides the modulus of elasticity $E$, the yield strength $\sigma_{\mathrm{y}}$, the ultimate tensile strength $\sigma_{\text {UTS }}$, the elongation at break and the normal $\bar{r}$ and planar $\Delta r$ anisotropy coefficients obtained from tensile test specimens cut out from the supplied sheets at $0^{\circ}, 45^{\circ}$ and $90^{\circ}$ with respect to the rolling direction (RD),

$\bar{r}=\frac{r_{0}+2 r_{45}+r_{90}}{4} \quad \Delta r=\frac{r_{0}-2 r_{45}+r_{90}}{2}$,

where $r_{0}, r_{45}$ and $r_{90}$ are the corresponding anisotropy coefficients at $0^{\circ}, 45^{\circ}$ and $90^{\circ}$.

\subsection{Characterization of Fracture Toughness}

The characterization of fracture toughness at room temperature was focused in opening mode I and made use of double-notched test specimens loaded in tension.

The specimens were cut out from the supplied aluminium AA1050-H111 sheets at $0^{\circ}, 45^{\circ}$ and $90^{\circ}$ degrees with respect to the rolling direction, and the tests were performed on an INSTRON 4507 universal testing machine in accordance with the methodology for determining the essential work of fracture that was originally proposed by Cotterell and Reddel [16].

The procedure used for determining fracture toughness in double-notched test specimens loaded in tension is summarized in Fig. 3. As seen in the Fig. 3, firstly the evolution of the tensile force with displacement is registered for a number of test cases performed with specimens having different lengths $c$ of the ligaments between the tips of the starter cracks (Fig. 3a). Secondly, the total energy $W$ is determined by integrating the evolution of the force with displacement until separation of the test specimen into two parts,

$W=\int_{0}^{x_{1}} F \mathrm{~d} x$,

where the symbol $x_{1}$ denotes the displacement $x$ at separation in case of a test specimen having a ligament with length $c_{1}$. The total energy $W$ corresponds to the grey area in Fig. 3b.

Thirdly, assuming the total energy $W$ to be split into the sum of a term associated with the energy $W_{\mathrm{p}}$ of plastic deformation and a term related to the energy $W_{\mathrm{s}}$ that is needed to form new surfaces at the tip of the cracks, where fracture takes place, the total energy per unit of area $w$ can be expressed as follows [13],

$w=\frac{W}{A}=\frac{W_{\mathrm{p}}}{A}+\frac{W_{\mathrm{s}}}{A}=\left(\bar{\sigma}_{\text {mean }} \bar{\varepsilon}_{\mathrm{av}}\right) \frac{\pi}{4} c+R$,

where $A=c \cdot t$ is the area of the ligament, $\bar{\sigma}_{\text {mean }}$ is the mean flow stress, and $\bar{\varepsilon}_{\mathrm{av}}$ is the final average value of the plastic strain in the cylindrical patch $V=\left(\pi c^{2} / 4\right) t$ where plastic deformation is confined between the notches (refer to the black circle in Fig. 3a). The symbol $R$ denotes the fracture toughness, which is defined as the amount of energy per unit of area that is required to create a new surface.

Finally, because the value of fracture toughness $R$ is difficult to isolate from $w$ in Eq. (7), the technique used for its determination involves extrapolating the energy per unit of area $w$ to the limiting conditions in which the length $c$ of the ligament approaches zero (Fig. 3c),

$R=\lim _{c \rightarrow 0}(w)$.

In graphical terms, Eq. (8) corresponds to the yintercept of a straight line with slope equal to $\alpha$ that contains the total energy per unit area $w$ of all the experiments performed with double-edge-notched test specimens having different lengths $c$ of the ligaments.

The application of the above described procedure for the characterization of fracture toughness of aluminium AA1050-H111 sheets with $1 \mathrm{~mm}$ thickness at room temperature is given in the following Sect. 4 .

\subsection{Formability Limits by Necking and Fracture}

The formability limits of the AA1050-H111 aluminium sheets by necking (FLC) were determined upon combination of the previously mentioned tensile tests with

Table 1 Summary of the mechanical properties of the AA1050-H111 aluminium sheets

\begin{tabular}{llllll}
\hline Orientation & $\begin{array}{l}\text { Modulus of } \\
\text { elasticity }(\mathrm{GPa})\end{array}$ & $\begin{array}{l}\text { Yield strength } \\
(\mathrm{MPa})\end{array}$ & $\begin{array}{l}\text { Ultimate tensile } \\
\text { strength (MPa) }\end{array}$ & $\begin{array}{l}\text { Elongation } \\
\text { at break }(\%)\end{array}$ & $\begin{array}{l}\text { Anisotropy } \\
\text { coefficient }\end{array}$ \\
\hline $0^{\circ} \mathrm{RD}$ & 72.7 & 115.4 & 119.0 & 7.1 & 0.71 \\
$45^{\circ} \mathrm{RD}$ & 67.9 & 120.4 & 121.2 & 5.2 & 0.88 \\
$90^{\circ} \mathrm{RD}$ & 71.8 & 123.0 & 120.8 & 5.6 & 0.87 \\
Average & 70.0 & 119.9 & 120.5 & 6.8 & 0.84 \\
\hline
\end{tabular}




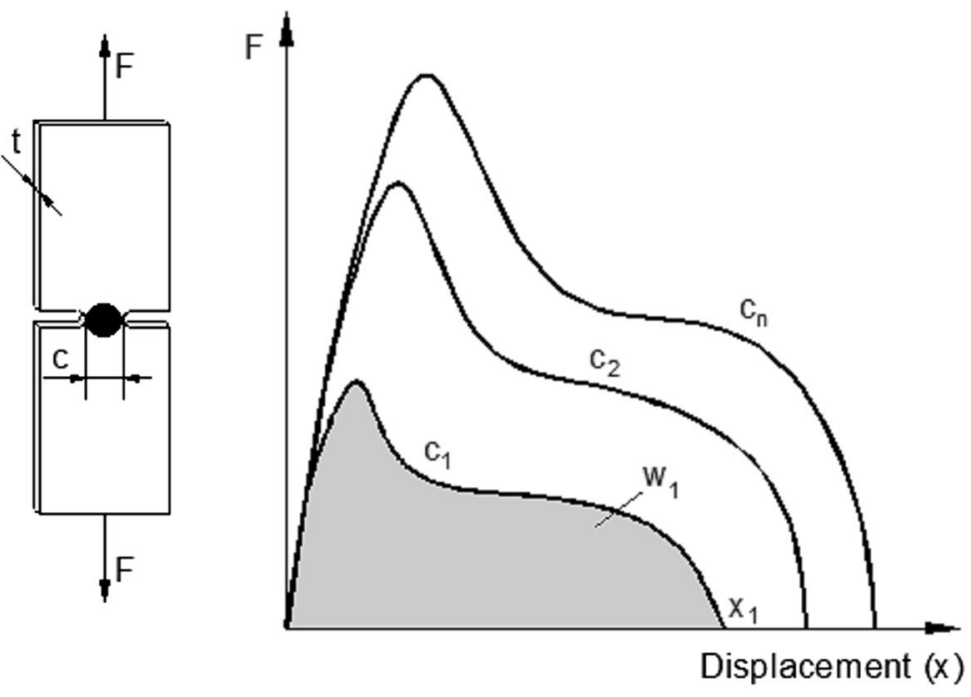

(a) (b)

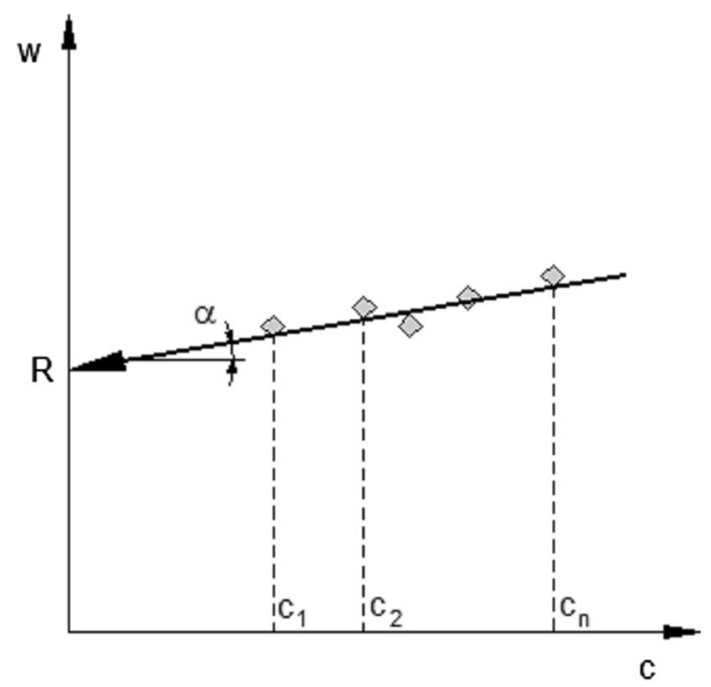

(c)

Fig. 3 Method and procedure used for determining fracture toughness $R$ : a schematic representation of a double-notched test specimen loaded in tension; $\mathbf{b}$ schematic evolution of the tensile force with displacement for test specimens with different lengths $c$ of the ligaments; $\mathbf{c}$ determining fracture toughness $R$ from extrapolation of the total energy per unit of area $w$

Nakajima, hemispherical dome and bulge tests. The Nakajima and hemispherical dome tests were performed in a flexible tool system that was installed in the INSTRON 4507 universal testing machine where the mechanical characterization of the material was carried out, whereas the circular and elliptical bulge tests were performed in an ERICHSEN 145/60 hydraulic universal testing machine.

The specimens utilized in the tests were electrochemical etched with a grid of overlapping circles with $2 \mathrm{~mm}$ initial diameter $d$ and the method employed for determining the FLC was based upon measuring the in-plane strains $\left(\varepsilon_{1}, \varepsilon_{2}\right)$ from grid points located along predefined directions crossing the crack perpendicularly. The in-plane strains $\left(\varepsilon_{1}, \varepsilon_{2}\right)$ at the grid points were obtained from conventional circle grid analysis,

$\varepsilon_{1}=\ln \left(\frac{l_{\mathrm{maj}}}{d}\right) \quad \varepsilon_{2}=\ln \left(\frac{l_{\mathrm{min}}}{d}\right)$,

where $l_{\text {maj }}$ and $l_{\min }$ are the lengths of the major and minor axes of the ellipses that resulted from plastic deformation of the original grid of overlapping circles during the tests.

The maximum strain pairs at the onset of necking were obtained after reconstructing the distribution of strains in the area of intense localization by means of a mathematical procedure that interpolates the experimental strains retrieved from adjacent deformed circles along a direction perpendicular to the crack by a parabolic 'bell-shaped curve'. The original procedure is described by Rossard [17] and evolved into the so-called 'position-dependent measurement' of the international standard for determination of FLCs [18]. The overall procedure is schematically described in Fig. 4a, and the resulting FLC is the ' $\mathrm{V}$-shaped' light grey curve in Fig. 4c.

The formability limits by fracture (FFL and SFFL) requires measuring the thickness of the specimens before and after fracture at several locations along the crack in order to obtain the 'gauge length' strains. The procedure is schematically described in Fig. 4b. The formability limits by fracture can be determined by means of the sheet formability tests that were utilized to determine the FLC, by means of double-notched test specimens loaded in tension, torsion and in-plane shear or by means of special purpose sheet metal forming processes such as single point incremental forming.

In the present investigation, the formability limits by fracture were determined by means of experiments performed with double-notched test specimens and single point incremental forming (SPIF) (Table 2).

The utilization of double-notched test specimens ensures a link with the testing procedures that are commonly employed to determine fracture toughness in fracture mechanics. The utilization of SPIF of simple truncated conical or pyramidal geometries with varying drawing angles allowed obtaining linear strain paths up to fracture. The results obtained with all these tests are given later in the paper. 


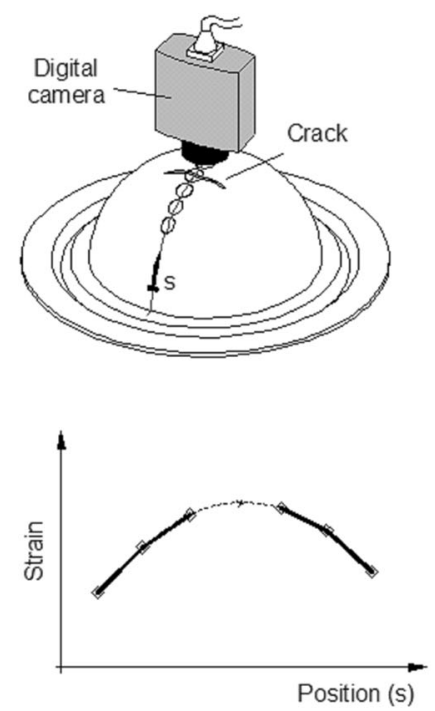

(a)

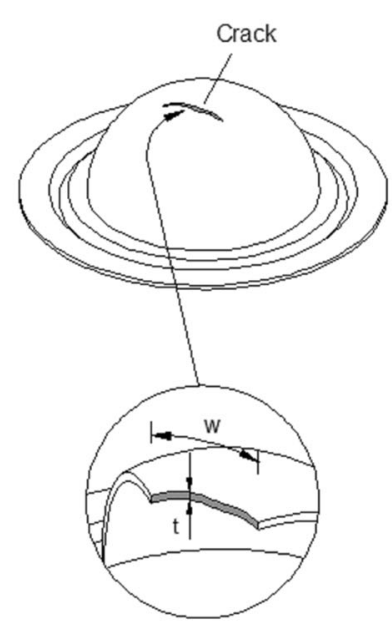

(b)

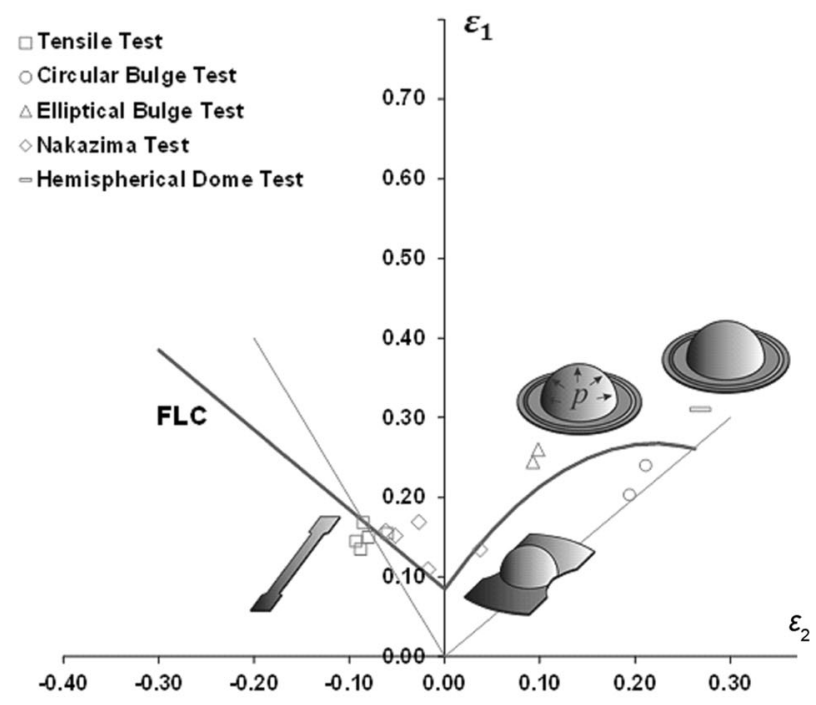

(c)

Fig. 4 Formability limits by necking and fracture: a schematic procedure to determine the in-plane strains at the onset of necking; $\mathbf{b}$ schematic procedure to determine the gauge length strains at the onset of fracture; $\mathbf{c}$ the FLC of the AA1050-H111 aluminium sheets with 1 mm thickness

Table 2 Experimental test specimens utilized in the characterization of the formability limits by fracture

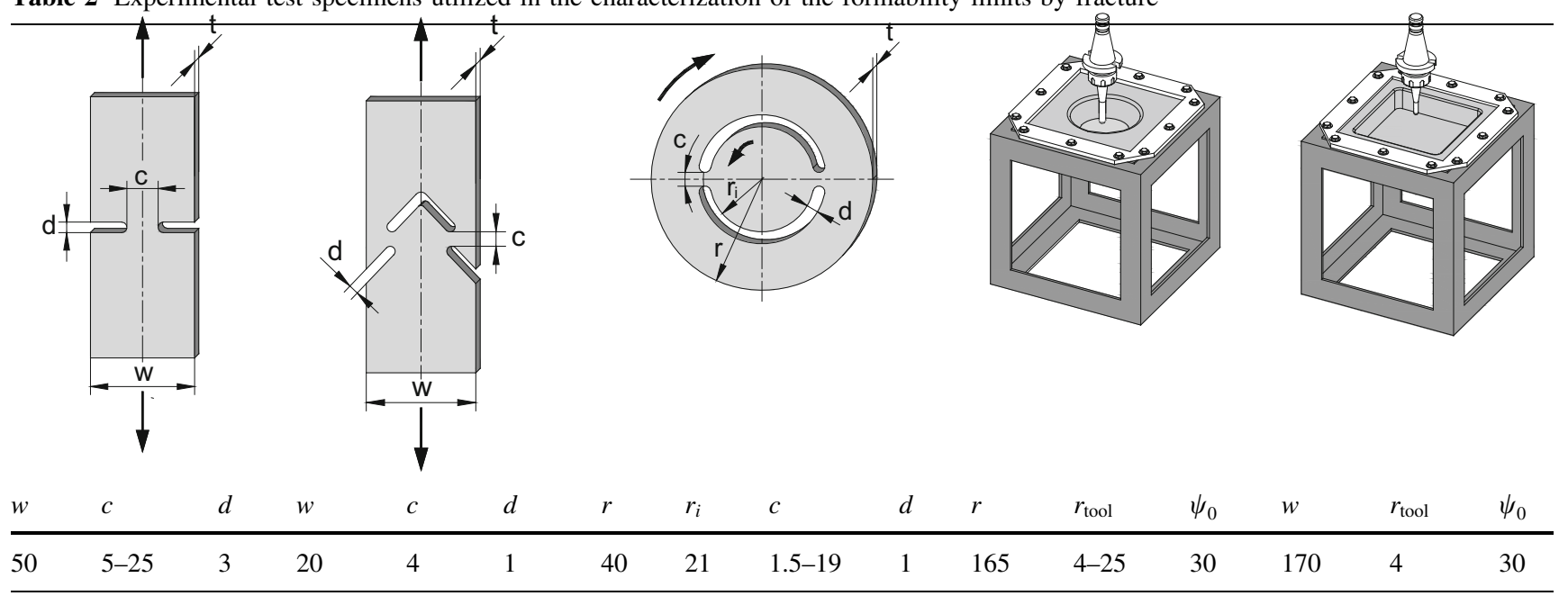

\section{Results and Discussion}

\subsection{Fracture Toughness and Crack Opening Mode I}

The determination of fracture toughness in crack opening mode I by means of double-edge-notched test specimens loaded in tension was performed in accordance with the procedure that was schematically depicted in Fig. 3. Consequently, by taking into consideration the experimental evolutions of the tensile force with displacement in doubleedge-notched test specimens with different ligaments $c$ of 5 , 10, 15, 20 and $25 \mathrm{~mm}$ that are shown in Fig. 5a. It is possible to conclude that the amount of energy per unit of area to create a new surface (fracture toughness) is equal to $R=56.87 \mathrm{~kJ} /$ $\mathrm{m}^{2}$. This value of fracture toughness $R=56.87 \mathrm{~kJ} / \mathrm{m}^{2}$ is an average value that results from double-edge-notched test specimens that were cut out from the supplied sheets at $0^{\circ}$ and $90^{\circ}$ with respect to the rolling direction.

The procedure employed for determining fracture toughness directly from truncated conical SPIF parts considers plastic work $W$ that makes up the specific work at fracture (also known as fracture toughness, $R$ ) to be dissipated in thin boundary layers of thickness $h$ alongside the crack surfaces (Fig. 6) [19], 

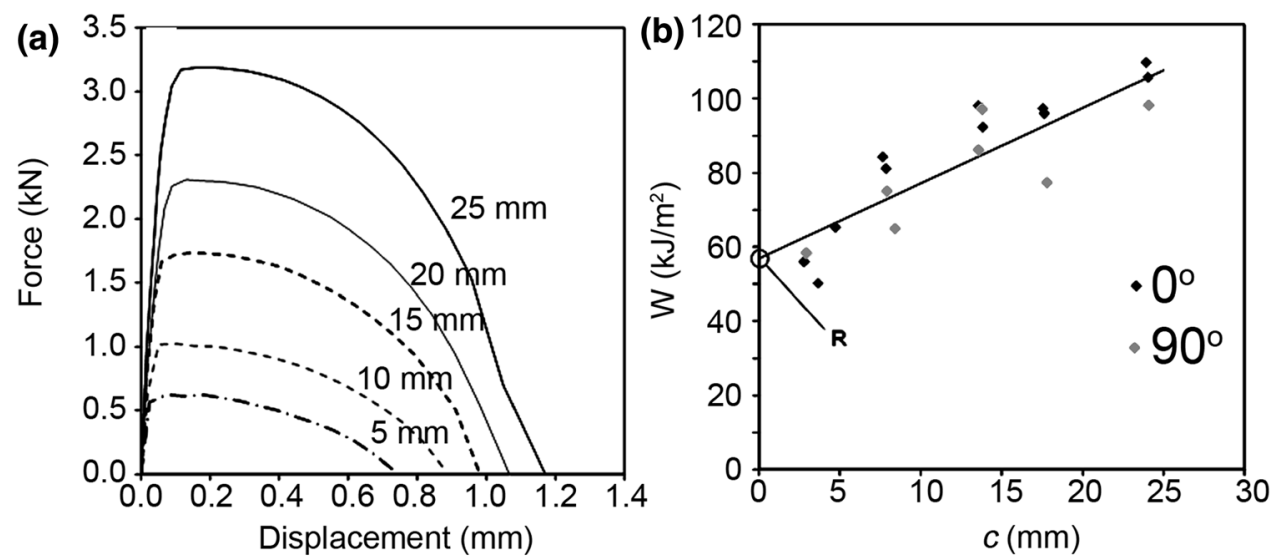

Fig. 5 Fracture toughness $R$ in AA1050-H111 aluminium sheets with $1 \mathrm{~mm}$ thickness obtained from double-edge-notched test specimens loaded in tension: a experimental evolution of the tensile force with displacement for test specimens with different ligaments $c$ that were cut out from the supplied sheets at $0^{\circ}$ with respect to the rolling direction; $\mathbf{b}$ average value of fracture toughness $R$ obtained from test specimens with different ligaments $c$ that were cut out from the supplied sheets at $0^{\circ}$ and $90^{\circ}$ with respect to the rolling direction

(a)

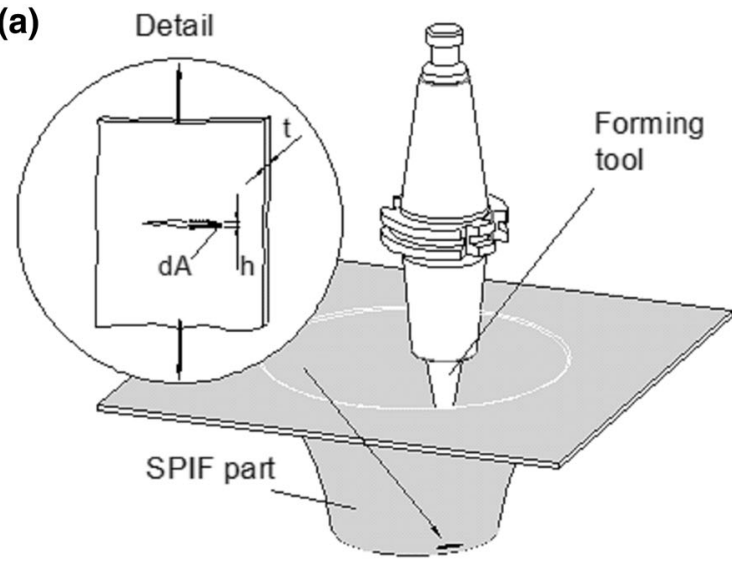

(b)

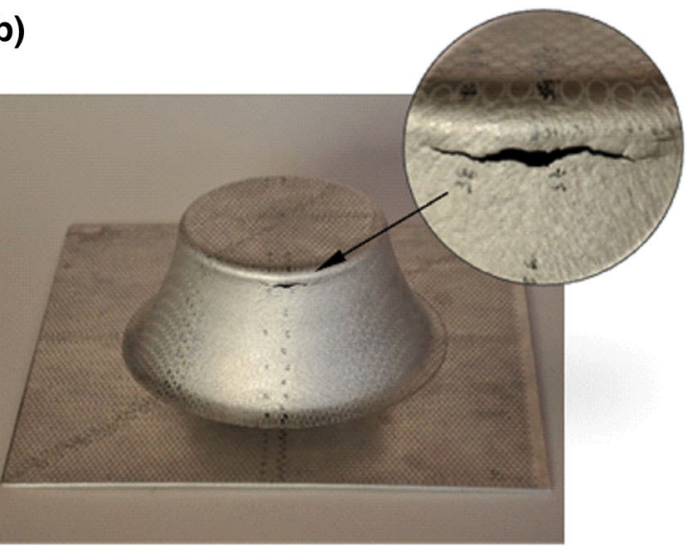

Fig. 6 Determining fracture toughness directly from SPIF tests: a circumferential crack with notation and detail showing the hatched region corresponding to a thin boundary layer alongside the crack; $\mathbf{b}$ truncated conical part fabricated by SPIF with a detail of a circumferential crack

$W=\left(\int_{0}^{\overline{\varepsilon_{\mathrm{f}}}} \bar{\sigma} \mathrm{d} \bar{\varepsilon}\right) h \mathrm{~d} A$,

where $\mathrm{d} A$ is the increase in crack area, $h \mathrm{~d} A$ is the associated increase in volume according to Atkins and Mai [13], $\bar{\sigma}$ is the effective stress, and $\bar{\varepsilon}$ is the effective strain.

The effective strain at fracture $\bar{\varepsilon}_{\mathrm{f}}$ is obtained from the experimental values of strain $\left(\varepsilon_{1 \mathrm{f}}, \varepsilon_{2 \mathrm{f}}, \varepsilon_{3 \mathrm{f}}\right)$ in the meridional, circumferential and thickness directions according to Hill's 1948 anisotropic yield criterion,

$\bar{\varepsilon}_{\mathrm{f}}=\frac{1+r}{\sqrt{(1+2 r)}} \sqrt{\varepsilon_{1 \mathrm{f}}^{2}+\varepsilon_{2 \mathrm{f}}^{2}+\frac{2 r}{(1+r)} \varepsilon_{1 \mathrm{f}} \varepsilon_{2 \mathrm{f}}}$.

Because fracture toughness $R$ is defined in Eq. (8) as the work per unit of area that is needed to create a new surface, its value can be determined by dividing the plastic work $W$ in Eq. (10) by the increase in crack area $\mathrm{d} A$ (refer once again to Fig. 6),

$R=h \int_{0}^{\overline{\varepsilon_{\mathrm{f}}}} \bar{\sigma} \mathrm{d} \bar{\varepsilon} \cong t \int_{0}^{\bar{\varepsilon}_{\bar{f}}} \bar{\sigma} \mathrm{d} \bar{\varepsilon}$,

where the approximation in Eq. (12) results from taking the thickness $h$ of the boundary layer as the deformed sheet thickness $t$ as it was suggested by Atkins and Mai [13] in their work on fracture toughness in sheet metal forming.

In physical terms, the assumption that the boundary layer $h$ alongside the crack surface is of the order of magnitude of the deformed sheet thickness $t$ is justified by the significant and uniform reduction of the initial sheet thickness $t_{0}$ (sometimes above $70 \%$ ) that is commonly observed in SPIF parts namely in truncated conical SPIF parts. 
Now, by taking into consideration that truncated conical SPIF parts undergo plastic deformation along proportional $\beta=\mathrm{d} \varepsilon_{1} / \mathrm{d} \varepsilon_{2}=\varepsilon_{1} / \varepsilon_{2}$, plane strain loading conditions (Fig. 7) and bearing in mind that the effective stress $\bar{\sigma}$ is calculated from the experimental values of the effective strain $\bar{\varepsilon}$ by means of Eq. (1), it is possible to determine fracture toughness $R$ directly from the experimental values of effective strain at fracture [refer to Eq. (11)], as follows,

$R \cong t \int_{0}^{\bar{\varepsilon}_{\mathrm{f}}} K \varepsilon^{n} \mathrm{~d} \bar{\varepsilon}=t \frac{K \bar{\varepsilon}_{\mathrm{f}}^{n+1}}{n+1}$.

The above equation provides a simple and effective procedure to determine fracture toughness $R$ from the black solid markers in Fig. 7 without the necessity of integrating the strains and stresses along the loading path. In fact, by replacing the effective strain $\bar{\varepsilon}_{\mathrm{f}}=1.64$ retrieved from the iso-effective strain contour plotted in Fig. 7 and the constant $K$ and the strain hardening exponent $n$ of the material stress-strain curve into Eq. (13), it is possible to determine an experimental value of fracture toughness $R=52.0 \mathrm{~kJ} / \mathrm{m}^{2}$.

The resemblance between the two aforementioned estimates of fracture toughness $\left(52.0\right.$ and $\left.56.87 \mathrm{~kJ} / \mathrm{m}^{2}\right)$ allows us to conclude that failure by fracture in truncated conical SPIF parts occurs by opening mode I (by tension) due to the key role played by the meridional stresses that are applied along the plastically deforming region resulting from the contact between the sheet and the forming tool. This conclusion is further justified by the circumstance that

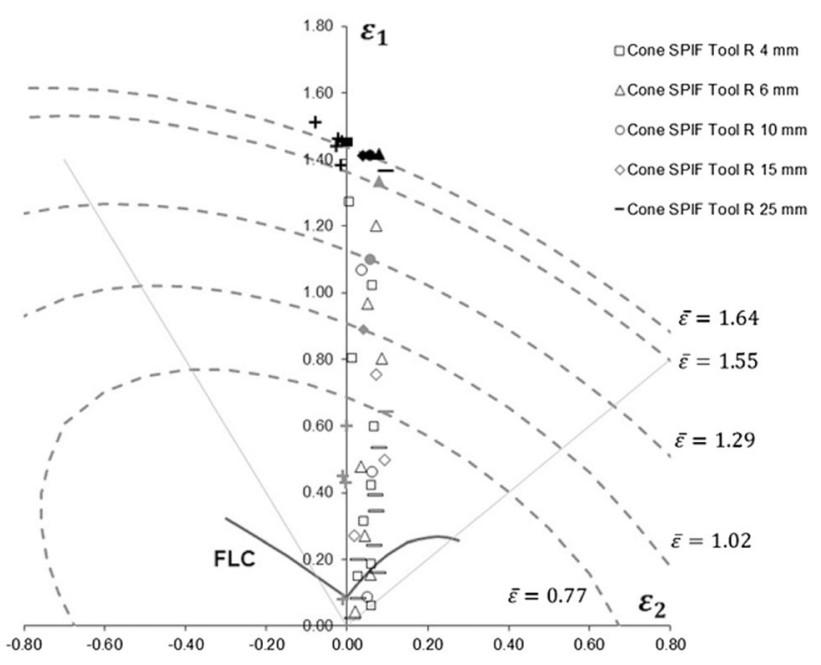

Fig. 7 Experimental strains obtained from measurements in truncated conical SPIF parts and double-notched test specimens loaded in tension. The grey solid markers refer to the strain pairs at the onset of necking, the black solid markers refer to the strain pairs at the onset of fracture, and the elliptical dashed grey curves refer to the iso-effective strain contours fracture strain pairs of the truncated conical parts that fail by circumferential cracking due to meridional tensile stresses being located very close to the fracture strain pairs of the double-notched test specimens loaded in tension that fail by cracking in opening mode I (Fig. 7). Later in the paper, it will be shown that the results of both tests lie on top of the FFL (fracture locus by tension) given by Eq. (2).

\subsection{Fracture Limits and Material Properties}

The FLCs are known to be dependent on material characteristics such as strain hardening, anisotropy and rate sensitivity as well as process operating conditions related to strain loading paths, amount of bending induced by tooling and sheet thickness. This implies that FLCs should not be considered a material property and, therefore, must be used with caution.

There are three other reasons that may stimulate researchers to consider the formability limits by fracture instead of the formability limits by necking. Firstly, the acceptance that engineers and technicians currently involved in the design of automotive sheet metal parts prefer to apply design guidelines based on the critical thickness reduction than on the forming limit curves (FLCs), in close agreement with the physics behind the definition of the FFL (refer to Sect. 2.1). Secondly, the well-known evidence that FLCs despite their simplicity and wide usage can fall short in the determination of the onset of necking due to difficulties in measurements. This often leads to the fact that FLCs of the same material provided by different sources may be different from each other. Thirdly, the understanding that currently available finite element programs that make use of ductile damage modelling for predicting the onset of failure require determination of the critical values of damage at the onset of fracture, in close agreement with the connection previously established between fracture limits, ductile damage and fracture toughness.

In order to better understand the advantage of using fracture limits instead of necking limits, let us consider the strain loading paths along the meridional direction of the truncated conical SPIF parts produced with different tool radius $r_{\text {tool }}$ that are plotted in Fig. 7 . The black solid markers correspond to fracture strain pairs obtained from gauge length strains and are independent from the radius $r_{\text {tool }}$ of the hemispherical-ended tools. The grey solid markers correspond to strain pairs that were obtained from in-plane strain measurements along predefined directions that cross the crack and were subsequently interpolated into a 'bell-shaped curve' in order to determine the maximum strains at the onset of necking. As seen in the figure, black and grey solid markers are coincident for the tests performed with hemispherical-ended tools of radius $r_{\text {tool }}$ of 4 
and $6 \mathrm{~mm}$ and are different for the remaining tests performed with hemispherical-ended tools of radius $r_{\text {tool }}$ of 10, 15 and $25 \mathrm{~mm}$. Moreover, the difference between black and grey solid markers increases with the $r_{\text {tool }}$.

The justification behind these results is directly related to the influence of the ratio $r_{\text {part }} / r_{\text {tool }}$ between the radius of the SPIF part $r_{\text {part }}$ and the radius of the hemisphericalended tool $r_{\text {tool }}$ on the physics of failure. In fact, large values of $r_{\text {part }} / r_{\text {tool }}$ and small tool radius $r_{\text {tool }}$ lead to failure by fracture with suppression of necking (meaning that black and grey solid markers are identical), whereas small values of $r_{\text {part }} / r_{\text {tool }}$ and large tool radius $r_{\text {tool }}$ lead to failure by fracture with previous necking (meaning that black and grey solid markers must be different). Moreover, results also show that the onset of failure by necking is delayed by the stabilizing effects induced by dynamic bending under tension that are controlled by the ratio $t / r_{\text {tool }}$ between the sheet thickness $t$ and the radius $r_{\text {tool }}$ of the forming tool.

All the above said allow concluding that fracture limits are not influenced by the amount of bending induced by the tool. Adding this conclusion to the aforementioned independency of the fracture limits from the strain loading paths (refer to Sect. 2), it follows that fracture limits can be considered a material property that only depends on sheet thickness. The dependency on sheet thickness makes sense from a fracture mechanics point of view because fracture toughness is known to experience changes with sheet thickness [20].

\subsection{Experimental Determination of the FFL and the SFFL}

The fracture loci of the AA1050-H111 aluminium sheets with $1 \mathrm{~mm}$ thickness are depicted in Fig. 8. The FFL was determined from the experimental fracture strain pairs obtained from the double-notched test specimens loaded in tension and the truncated conical and pyramidal SPIF parts that are listed in Table 2. All these fracture strain pairs prove the relation between the FFL and crack opening by tension (mode I). The SFFL was determined from the experimental fracture strain pairs obtained from the torsion and in-plane shear tests that are also listed in Table 2.

The strain loading paths of the SPIF parts were determined from circle grid analysis, whereas the strain loading paths of the double-notched test specimens were determined by means of a digital image correlation system (Aramis from GOM mbH).

The interpolation of the fracture strain pairs depicted in Fig. 8 provides the following results for the FFL and SFFL of aluminium AA1050-H111 sheets with $1 \mathrm{~mm}$ thickness,

$\varepsilon_{1 \mathrm{f}}+0.86 \varepsilon_{2 \mathrm{f}}=1.40(\mathrm{FFL})$,

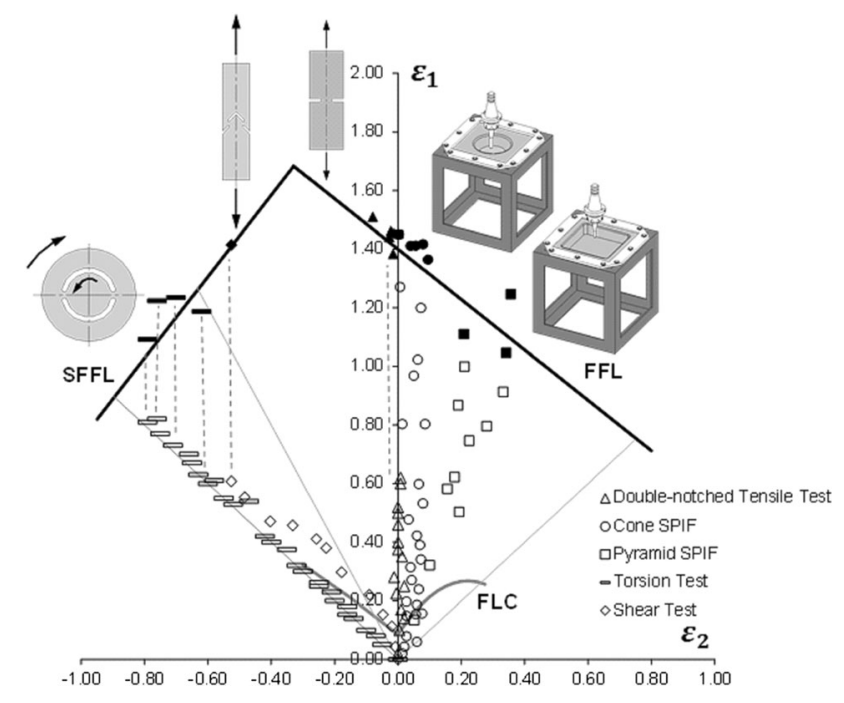

Fig. 8 Experimental fracture strain pairs obtained from the tests listed in Table 2 that were utilized to determine the fracture loci of the AA1050-H111 aluminium sheets with $1 \mathrm{~mm}$ thickness

and

$\varepsilon_{1 \mathrm{f}}-1.39 \varepsilon_{2 \mathrm{f}}=2.14(\mathrm{SFFL})$.

The slopes of the interpolated lines are in fair agreement with the theoretical slopes equal to -1 and +1 that were predicted by Eqs. (2) and (3) but the resulting angle between them $\left(\sim 85^{\circ}\right)$ is in excellent agreement with the condition of perpendicularity between the two fracture lines, under the assumption of no development of mixedcrack separation modes.

The fact that the slopes of the FFL and the SFFL are perpendicular, yet they are different from +1 and -1 suggests that there is a missing link in the proposed analytical framework that ought to make the slopes dependent on some other effects that are not included in the approach such as, coupled ductile damage, the existence of a threshold strain $\bar{\varepsilon}_{0}$ below which damage is not accumulated and the utilization of other yield criteria than that in Hill's work [21] that are more appropriate for modelling plastic flow of aluminium alloys, among others. This requires future research work.

\section{Conclusions}

This paper presents a new vision behind the formability limits by fracture that makes use of fundamental concepts of plasticity theory, ductile damage and fracture mechanics. The fracture by tension is associated with mode I of fracture mechanics and to the physical failure mechanism of excessive thinning. The fracture by in-plane shear is associated with mode II of fracture mechanics and to the 
physical failure mechanism of excessive in-plane distortion.

Experiments with double-notched test specimens loaded in tension, torsion and in-plane shear, and truncated conical and pyramidal parts produced by SPIF proved effective to determine the fracture loci of AA1050-H111 aluminium sheets with $1 \mathrm{~mm}$ thickness.

The independence of fracture loci from strain loading paths that is intrinsic to the analytical framework and the lack of sensitivity to bending demonstrated via experimental measurement of strains in truncated conical parts produced by SPIF help understanding the reason why fracture loci (FFLs and SFFLs), instead of FLCs, should be considered material properties.

Acknowledgments M.B. Silva and P.A.F. Martins would like to acknowledge the support provided by Fundação para a Ciência e a Tecnologia of Portugal within project LAETA-UID/EMS/50022/ 2013 and SFRH/BSAB/105959/2015. K. Isik and A. E. Tekkaya gratefully acknowledge funding by the German Research Foundation (DFG) within the scope of the Transregional Collaborative Research Centre on sheet-bulk metal forming (SFB/TR 73) in the subproject C4 'Analysis of load history dependent evolution of damage and microstructure for the numerical design of sheet-bulk metal forming processes'.

\section{References}

[1] Z. Marciniak, Adv. Technol. Plast. 1, 685 (1984)

[2] J.B. Kim, D.Y. Yang, Eng. Comput. 20, 6 (2003)

[3] S.P. Keeler, Circular Grid System-A Valuable Aid for Evaluating Sheet Metal Formability. SAE technical paper 680092 (1968)
[4] G. Goodwin, Application of Strain Analysis to Sheet Metal Forming Problems in the Press Shop. SAE technical paper 68009 (1968)

[5] H.W. Swift, J. Mech. Phys. Solids 1, 1 (1952)

[6] R. Hill, J. Mechan, Phys. Solids 1, 19 (1952)

[7] Z. Marciniak, K. Kuckzynski, Int. J. Mech. Sci. 9, 609 (1967)

[8] A.G. Atkins, J. Mater. Process. Technol. 56, 609 (1996)

[9] F.A. McClintock, J. Appl. Mech. 35, 363 (1968)

[10] C.M. Muscat-Fenech, S. Arndt, A.G. Atkins, in Proceedings of the 4th International Conference, University of Twente, The Nederlands, 1996, pp. 249-260

[11] T. Wierzbicki, Y. Bao, Y.W. Lee, Y. Bai, Int. J. Mech. Sci. 47, 719 (2005)

[12] K. Isik, M.B. Silva, A.E. Tekkaya, P.A.F. Martins, J. Mater. Process. Technol. 214, 1557 (2014)

[13] A.G. Atkins, Y.W. Mai, Elastic and Plastic Fracture: Metals, Polymers, Ceramics, Composites, Biological Materials (EllisHorwood, Chichester, 1975)

[14] P.A.F. Martins, N. Bay, A.E. Tekkaya, A.G. Atkins, Int. J. Mech. Sci. 83, 112 (2014)

[15] ASTM Standard E8/E8 M, Standard Test Methods for Tension Testing of Metallic Materials (ASTM International, West Conshohocken, USA, 2013)

[16] B. Cotterell, J.K. Reddel, Int. J. Fract. 13, 267 (1977)

[17] C. Rossard, Mise en forme des métaux et alliages (CNRS, Paris, 1976)

[18] ISO Standard 12004-2, Metallic Materials—Sheet and StripDetermination of Forming-limit Curves-Part 2: Determination of Forming-Limit Curves in the Laboratory, Geneva, Switzerland, 2008

[19] V. Cristino, L. Montanari, M.B. Silva, A.G. Atkins, P.A.F. Martins, Int. J. Mech. Sci. 83, 146 (2014)

[20] A.G. Atkins, P.A.F. Martins, Thin Sheet Fracture, in Materials Science and Materials Engineering (Elsevier, UK, 2016)

[21] R. Hill, Proc. R. Soc. Lond. A 193, 281 (1948) 